Gynäk. Rdsch. 1981;21:I-VIII

\title{
Contents, Vol. 21, 1981
}

\section{Nr.1}

Mammakarzinom: Neue Aspekte für die Praxis

Einleitung

Brunner, K.W 5

Die brusterhaltende Strahlentherapie beim operablen Mammakarzinom

Veraguth, $\mathrm{P}$ 8

Hormonrezeptoren und Mammakarzinom

Goldhirsch, A.; Leuenberger, U.; Cavalli, F 18

Postoperative Chemoprophylaxe beim operablen Mammakarzinom

Brunner, K.W 24

Problematik der brusterhaltenden Operation beim Mammakarzinom

Nöthiger, F 34

Bisherige Resultate und allfállige Indikationen der brusterhaltenden Therapie

Harder, F 40

Vorbehalte zu den brusterhaltenden Operationen

Berger, M 46

Mammakarzinom: Methoden zur Erkennung prognostischer Faktoren

Zimmermann, A 56

Probleme der adjuvanten Chemotherapie und Rezeptorenbestimmung aus der Sicht der Praxis

Kammer, G 65

Ankündigung 68

$\mathrm{Nr} .2$

Rundgespräch · Table ronde

Sinn und Unsinn von Hormonbestimmungen: Tagung der Schweizerischen Gesellschaft für Gynäkologie

und Geburtshilfe, St. Gallen, 18.-20. 6. 1980

Kubli, F 69

Anorexie nerveuse

Herrmann, W.; Bourrit, B 75

Sinn und Unsinn von Hormonbestimmungen in Gynäkologie und Geburtshilfe. Sekundäre

Amenorrhö,

Androgenisierung, Sterilität

Hammerstein, J 78

Hormonbestimmungen während der Perimenopause

Plotz, E.J 86

Sinn und Unsinn der Hormonbestimmungen in Geburtshilfe und Gynäkologie:

Spätschwangerschaft

Keller, P.J 89

Hormonale Diagnostik in der Frühgravidität 
Runnebaum, B.; Gerhard, 194

IV Inhaltsverzeichnis

Originalarbeiten

Klinisch-chemische und hämatologische Parameter im Schwangerschaftsverlauf

(Clinicochemical and

Haematological Parameters in the Course of Pregnancy)

Brockerhoff, P.; Kurtenbach, M.I.; Stark, W.; Schicketanz, K.H.; Friedberg, V.; Rathgen, G.H.

101 Erfahrungen mit einer vereinfachten Form des Roll-over-Tests (Experience with a

Simplified Form of the

Roll-Over Test)

Huber, J.C.; Reinold, E

124

Zervix- und Vaginalzytologie unter IUD-Prophylaxe (mit 1 Farbtafel) (Cervical and Vaginal

Cytology in

Women with IUDs) (with 1 color plate)

Grumbrecht, C 131

Kurzfassungen von Zeitschriftenartikeln

KJinische Erfahrungen mit den antepartalen CTG-Auswerteverfahren nach Fischer, Hammacher, Hodr

und Kubli

Wilken, H.P.; Hackl, P.; Wilken, H 135

Der Einfluss des Zigarettenrauchens der Mutter auf das Geburtsgewicht und auf die Zunahme des fetalen

biparietalen Durchmessers

Murphy, J.F.; Drumm, J.E.; Mulcahy, R

Beherrschung perinataler Infektionen durch herkömmliche Vorbeugungsmassnahmen

Iffy, L.; Kaminetzky, H.A.; Maidman, J.E.; Lindsey, J.; Arrata, S.M 138

Nr.3

Originalarbeiten

Schwangerschaft und Immunosuppressiva (Pregnancy and Immunosuppressive Agents)

Fischer, B. von; Gigon, U.; Sidiropoulos, D.; Montandon, A 141

Psychologische Aspekte der Kontrazeption mit dem Multiload Cu250 (Psychological Aspects of

Contra

ception Using Multiload Cu250)

Rauchfleisch, U 159

Virusisolierung im Fruchtwasser bei Infektion in der Friihschwangerschaft (Isolation of Virus in the

Amniotic Fluid in Infections of Early Pregnancy)

Schilt, U.; Sidiropoulos, D 166

Kurzfassungen von Zeitschriftenartikeln

Eingeschränkte Bedeutung des LRH-Tests in der klinischen Routinediagnostik

Böhm, P.; Häffele, R 171

Budd-Chiari-Syndrom nach Einnahme von Ovulationshemmern

Gstöttner, G.; Rothe, K.; Leitsmann, H.; Langpeter, D 172

Behandlung des Asherman-Syndroms

Smid, I.; Borsos, A 173

Zur Wertigkeit von Immunkontrollen bei Immuntherapie gynäkologischer Karzinome 
Disaia, P.J.; Rich, W.M

175.

Verstärkung der Leukozytenmigration als Ausdruck gesteigerter immunologischer Reaktivität Gleicher, N.; Beers, P.; Kerenyi, T.D.; Cohen, C.J.; Gusberg, S.B 176

Serumspiegel von schwangerschaftsspezifischem Glykoprotein in der Spätschwangerschaft:

Tagesschwan-

kung, Schwankung unter Wehentätigkeit

Grudzinskas, J.G.; Obiekwe, B.C.; Frumar, A.M.; Hartmann, K.G.; Schlesinger, P.; Chard, T. . 178

C. Grumbrecht

Farbtafel I

1

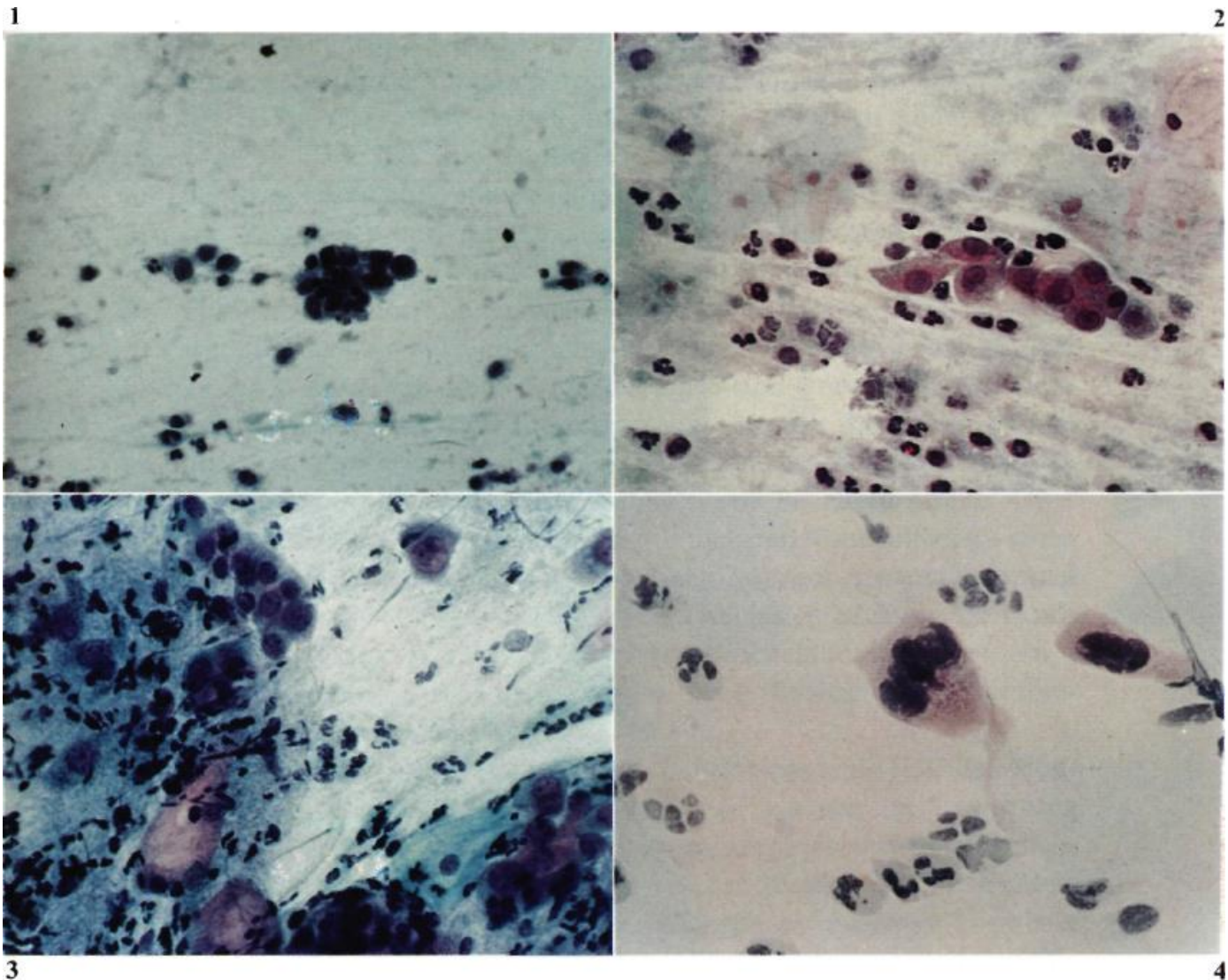

Abb. 1. Gruppe von Endometriumzellen. 26jährige Patientin ohne Beschwerden, seit 9 Monaten CU-T.

Abb. 2. Unruhige Metaplasie. 35jährige Patientin, seit 4 Monaten ML 250, klinisch beschwerdefrei.

Abb. 3. Endozervizitis. Unruhige Metaplasie der endozervikalen Epithelien, Hyper-chromasie und Ballonierung des Karioplasma, Pseudoeosinophilie des Zytoplasma. 34jäh-rige Patientin, seit 2 Monaten CU-7, klinisch massiver Fluor.

Abb. 4. Zervixepithelien mit stark verformten hyperchromen Kernen. 29jährige Patientin, seit 7 Monaten CU-T. Klinisch mässiger Fluor. Entfernung des IUD, nach 3 Monaten zytologisch unauñalliger Abstrich.

Gynäkologische Rundschau, Vol. 21 


\section{S. Karger, Basel \\ Inhaltsverzeichnis \\ $\mathrm{V}$}

Der Ursprung der Peritonealflüssigkeit der Frau: ein Exsudat aus den Ovarien

Konickx, P.R.; Renaer, M.; Brosens, I.A 179

Verwendung des Foley-Katheters zur Behandlung der unreifen Zervix vor der Geburtseinleitung

Ezimokhai, M.; Nwabineli, J.N 180

Die Brustuntersuchung führt nicht zur Erhöhung des Serumprolaktins

Jarrell, J.; Franks, S.; Mclnnes, R.; Gemayel, K.; Guyda, H.; Arronet, G.H.; Naftolin, F 182

Hypophysen- und Ovarfunktion bei Frauen mit hormonaler Kontrazeption

Cohen, B.L.; Katz, M 183

Fortbildung

II. Winterfortbildungstagung der Osterreichischen Gesellschaft für Gynäkologie und

Geburtshilfe, Obergurgl, 3.-9. Februar 1980. Kurzfassungen der Referate

Die organisatorischen Entwicklungstendenzen in Geburtshilfe und Gynäkologie

Gitsch, E 185

Klinik und Therapie der Entzündungen des inneren Genitales

Gitsch, E $\quad 186$

Kolposkopie

Burghardt, E 187

Biochemische Aspekte des Krebsproblems

Schauenstein, E.; Schaur, R.J 189

Tumorimmunologie: Stand fur die praktische Medizin und deren diagnostische und therapeutische Kon-

sequenzen

Tilz, G.P.; Becker, H 191

Hormontherapie in der Praxis. Einführung zum Thema

Janisch, H 194

Fortschritte in der Diagnostik und Therapie männlicher Fertilitätsstörungen

Bartsch, G.; Scheiber, K 196

Ankündigungen 199

Nr.4

Originalarbeiten

Effizienz psychologischer Verfahren zur Entbindungsvorbereitung. Eine feldexperimentelle

Studie (Effi

cacy of Psychological Methods to Prepare Childbirth. A Field-Experimental Study)

Borlinghaus, K; Hülter, K 201

Rauchen und Schwangerschaft (Smoking and Pregnancy)

Kunze, M.; Schoberberger, R.; Gredler, B 213

Kurzfassungen von Zeitschriftenartikeln

Schwankungen des Plasma-LRH während des normalen Zyklus

Miyake, A.; Kawamura, Y.; Aono, T.; Kurachi, K 223

Maturation et fécondation in vitro d'ovocytes humains

Cohen, J.; Mandelbaum, J.; Plachot, M 224

VI

Inhaltsverzeichnis 
Zirkadiane Schwankungen der Plasmaspiegel von luteinisierendem Hormon (LH),

follikelstimulierendem

Hormon (FSH) und Prolaktin (PRL) bei präpubertären und pubertären Knaben und Mädchen

Beck, W.; Wuttke, W 225

Östrogentherapie und Mammakarzinom

Völker, W.; Kannengiesser, U.; Majewski, A

Adjuvante Chemotherapie mit Trofosfamid, Methotrexat und Fluorourazil beim

Mammakarzinom

Albrecht, M.; Jepsen, G.; Trams, G.; Thomson, K 229

Therapie der postoperativen Harnverhaltung mit Prostaglandin

Griinberger, W.; Tulzer, H 231

Erste Ergebnisse zur extrakorporalen Befruchtung am Menschen

Mettler, L.; Seki, M.; Baukloh, V.; Semm, K 233

Zur Therapie der Gestosen

Friedberg, V 234

Tumormarker und ihre Bedeutung in der gynäkologischen Onkologie

Melchert, F.; Kreienberg, R 238

Östrogenrezeptoren in normalen und myomatösen Uteri

Büchi, K.; Keller, P.J 239

Das Korpuskarzinom - eine Studie mit zahlreichen Parametern

Cauchi, M.N.; Goriup, D.; Riglar, C; Quinn, M.A.; Richardson, C.R 240

Eine einfache Technik zur Steigerung der Erfolgsrate von Inseminationen

Makler, A 242

Verstärkte und verlängerte Freisetzung von LH und FSH nach intravenöser Verabreichung eines synthe-

tischen LH-RH-Analogons (E > -Ser [TBU]6-EA10-LH-RH) bei gesunden Männern

verschiedenen

Alters

Vierhapper, H.; Waldhäusl, W

243

Scheidenrekonstruktion in der gynäkologischen Onkologie: Ein Überblick über

Operationsverfahren

Magrina, J.F.; Masterson, B.J 245

Mykoplasmen und Ureaplasmen bei Infertilität und Abortus

Friberg, J 247

Beckenadhäsionen nach mikro- und makrochirurgischer Keilsektion der Ovarien

Eddy, C.A.; Asch, R.H.; Blamaceda, J.P 249

Plasma-Prostaglandin-Konzentrationen nach Cerclage in der Frühschwangerschaft

Toplis, P.J.; Shepherd, J.H.; Youssefmejadian, E.; Jankubowicz, D.; Dewhurst, Sir J

Androgene und Ostrogene im Serum von Frauen nach der Menopause mit bzw. ohne

Korpuskarzinom

Judd, H.L.; Davidson, B.J.; Frumar, A.M.; Shamonki, I.M.; Lagasse, L.D.; Ballon, S.C

Endometriumkarzinom in Stadium I

Malkasian, G.D.; Annegers, J.F.; Fountain, K.S . 253

Zur Unterscheidung Lymphknotenmetastasen - gutartige drüsige Einschlüsse bei

Ovarialkarzinomen mit

niedriger Malignität 
Ehrmann, R.L.; Federschneider, J.M.; Knapp, R.C 255

Risiko von Östrogenbehandlungen in bezug auf das Endometriumkarzinom

Jelovsek, F.R.; Hammond, C.B.; Woodard, B.H.; Draffin, R..; Lee, K.L.; Creasman, W.T.;

Parker,

R.T 257

Die Wertigkeit einer adjuvanten Therapie beim Ovarialkarzinom des Stadiums I

Hreshchyshyn, M.M.; Park, R.C; Blessing, J.A.; Norris, H.J.; Lagasse, L.D.; Creasman, W.T. .

259 Farblymphographische Ergebnisse mit einer Kohlenstoff-Lipidol-Suspension bei

Karzinomen des

weiblichen Genitales

Fröhlich, H.H.; Schmidt, F.; Piskazeck, K; Krieg, U 260

Kolpopoese aus dem Becken-Peritoneum

Kurbanova, A.G 262

Inhaltsverzeichnis VII

Gewinnung menschlicher Eizellen

Weise, W 263

Radikalitätsbestimmung der iliakalen Lymphonodektomie im Rahmen der Wertheim-

Radikalopera-

tion

Leitsmann, H.; Pawlowitsch, T.; Bilek, K.; Friedrichs, G 264

Die atypische Hyperplasie des Endometnums - ihre prognostische Tendenz und Therapie

Mühlnickel, D.; Link, M 266

Fortbildung

«lntimhygiene der Frau» im Collegium publicum gynaecologicum der I. Universitäts-

Frauenklinik Wien am 20. Juni 1980

Zur Intimpflege der äusseren Genitalregion

Gitsch, E 268

Die Hygiene der Frau während der Menstruation und nach gynäkologischen Operationen

Philipp, K 271

Hygiene in Schwangerschaft und Wochenbett

Kubista, E 273

Hygiene nach Strahlentherapie

Kucera, H 275

Das Ovarialkarzinom

Vorwort

Gitsch, E 277

Zur Chirurgie des Ovarialkarzinoms

Gitsch, E 278

Strahlentherapie des Ovarialkarzinoms

Kärcher, K.H 284

Zur Chemotherapie der Ovarialkarzinome

Schmidt, C.G 286

Erratum 290

Autorenindex 291

Supplementum 1

Schweizerische Gesellschaft fur Senologie 
Kongress in Zurich, 16. und 17. November 1979

Société Suisse de Senologie

Congrès à Zurich, 16 et 17 novembre 1979

VIII Inhaltsverzeichnis

Supplementum 2

Jahrestagung der Bayenschen Gesellschaft für Geburtshilfe und Frauenheilkunde und der Osterreichischen Gesellschaft für Gynäkologie und Geburtshilfe

Juni 1981, München

Supplementum 3

Schweizerische Gesellschaft für Gynäkologie

Bericht über die Jahresversammlung, 11. bis 13. Juni 1981 in Genf

Société Suisse de Gynécologie

Comptes rendus de $\Gamma$ Assemblée annuelle, 11 au 13 juin 1981 à Geneve 